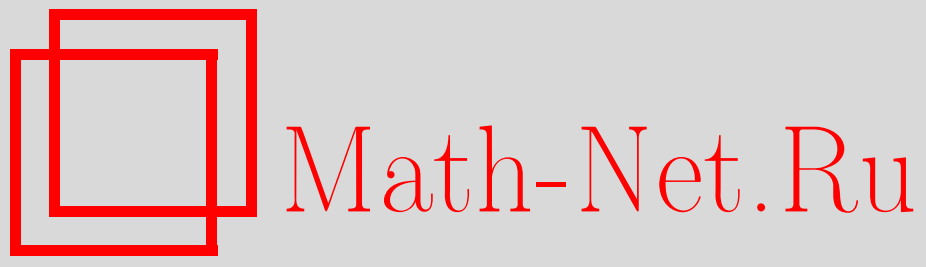

В. А. Клепцын, М. Б. Нальский, Сближение орбит в случайных динамических системах на окружности, Функи. анализ и его прил., 2004, том 38, выпуск $4,36-54$

DOI: https://doi.org/10.4213/faa125

Использование Общероссийского математического портала MathNet.Ru подразумевает, что вы прочитали и согласны с пользовательским соглашением

http://www. mathnet.ru/rus/agreement

Параметры загрузки:

IP : 18.208 .226 .222

26 апреля 2023 г., 13:01:12

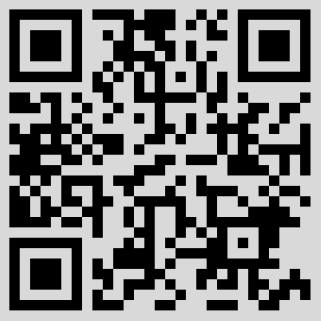




\title{
Сближение орбит в случайных динамических системах на окружности*
}

\author{
(c) 2004. В. А. КлЕПЦЫН, М. Б. НАЛЬСКИЙ
}

\section{§1. Введение}

1.1. Случайные динамические системы на окружности. Пусть задано конечное число сохраняющих ориентацию гомеоморфизмов $T_{i}$ окружности. Последовательность итераций порожденной ими случайной динамической системы - это случайная последовательность гомеоморфизмов, начинающаяся с тождественного отображения, в которой каждый следующий элемент получается из предыдущего применением одного из $T_{i}$, выбираемого случайно и независимо на разных шагах. Точное определение будет дано ниже, в разд. 1.3.

Компьютерное моделирование случайных динамических систем на окружности, проведенное С. И. Трифоновым и М. Б. Нальским, показало следующий эффект, наблюдающийся во многих сходных ситуациях (см. [1,4]): при увеличении числа итераций расстояние между образами различных точек на окружности стремилось к 0. В предлагаемой работе этот эффект будет обоснован теоретически при некоторых предположениях на гомеоморфизмы. Этим предположениям, как будет показано, удовлетворяет (в частности) некоторое открытое в $C^{1}$-топологии множество в пространстве случайных динамических систем.

Случайные динамические системы на окружности исследовались многими авторами. Кайзер [1] рассматривал итерации аналитического диффеоморфизма, между которыми происходит поворот на случайный угол. В предположениях типа общности положения, наложенных на диффеоморфизм и на распределение значений угла поворота, он доказал, что расстояние между траекториями стремится к 0 почти наверное.

Кроме того, случайная динамическая система может быть интерпретирована как пара, состоящая из случайного блуждания по (полу)группе и действия этой (полу)группы на фазовом пространстве (в данном случае окружности). Исследование такой пары (блуждание, действие) тесно связано с задачей описания границы Пуассона случайного блуждания. Это обуславливает сходство методов, применяемых при исследовании границ Пуассона и случайных динамических систем. В частности, сходимость итераций стационарной меры к $\delta$-мере, применяемая в данной статье - важный инструмент для описания границы Пуассона случайного блуждания; см. [8]. Кроме того, доказательство единственности стационарной меры, проводимое в данной статье, аналогично доказательству единственности стационарной меры, проводимому в [8].

Случайные динамические системы на сфере, полученные проективизацией линейных отображений пространства $\mathbb{R}^{n}$, тесно связаны с исследованием пока-

* Работа осуществлена при частичной поддержке грантов РФФИ 02-01-00482, РФФИ 0201-22002 и CRDF RM1-2358-MO-02. 
зателей Ляпунова произведений случайных матриц. Например, наличие одного показателя Ляпунова, большего остальных, означает, что длинная композиция линейных отображений сильно растягивает вдоль некоторого (случайного) направления, а следовательно, соответствующая композиция проективных отображений переводит бо́льшую часть пространства $\mathbb{R} P^{n}$ в малую окрестность одной (случайной) точки.

Такие системы исследовались многими авторами: Фюрстенбергом [4,6], Ле Жаном [3], Кестеном [6], Кифером [7], Крауэлом [9]. Теорема 8.3 работы [4] утверждает, что если система проективных отображений неприводима, то для обращенного порядка итераций расстояние между образами точек почти наверное стремится к 0 (в любой размерности). Это, в частности, означает, что в одномерном случае - проективная динамика на окружности - траектории сближаются и для обычного порядка итераций.

Показатели Ляпунова случайных динамических систем на многообразиях исследовались также в работе Баксендейла [13]. В ней показывается, что для любой гладкой случайной динамической системы либо найдется мера, инвариантная относительно всех отображений системы (случай, почти никогда не реализующийся), либо для некоторой эргодической стационарной меры сумма соответствующих ей показателей Ляпунова будет строго отрицательной. В случае $C^{1+\varepsilon}$-гладкой динамики на окружности отсюда можно вывести локальную версию теоремы 1 настоящей работы: для каждой точки $x \in S^{1}$ почти наверное найдется окрестность $U \ni x$, такая, что орбиты точек из $U$ сближаются с орбитой точки $x$.

Настоящая статья содержит технику, позволяющую рассматривать случай конечного числа гомеоморфизмов (и не очень сложно обобщаемую на случай произвольного семейства порождающих отображений) даже без предположения об их гладкости. K сожалению, платой за это является невозможность применения этой техники для исследования показателей Ляпунова в случае гладких систем (т. е. к решению вопроса об экспоненциальности скорости сжатия). Кроме того, эта техника может применяться и в некоторых других ситуациях (см. §7) - правда, давая уже известные результаты.

Использованная в настоящей работе идея обращения порядка итераций содержится в работах Крауэла [9], Ле Жана [3], Фюрстенберга [4,5], Каймановича и Мазура [8]. Понятия мартингала и марковского момента, а также теорему о сходимости мартингалов, тоже использующиеся здесь, можно найти, например, в книге Ширяева [2].

1.2. Структура статьи. В разд. 1.3 вводятся обозначения и даются необходимые определения. В $§ 2$ сформулированы основные результаты работы (разд. 2.1), некоторые их обобщения (приводимые без доказательства, разд. 2.3), а также следствия для косых произведений со слоем - окружностью (разд. 2.2). В разд. 2.4 описана схема доказательства основных результатов, а в $\S 3-5$ они доказываются. В 66 приведен пример (принадлежащий Городецкому и Ильяшенко $[10,11]) C^{1}$-открытой области в пространстве систем, в которой имеет место описанный эффект сближения орбит. В $§ 7$ исследуется, в каких еще случаях, кроме одномерной динамики, применима техника настоящей работы. Наконец, в §8 сформулированы некоторые открытые вопросы.

Благодарности. Авторы хотели бы выразить свою глубокую благодарность Ю. С. Ильяшенко и А. С. Городецкому за постановку задачи, за многократные дискуссии и помощь в подготовке настоящей работы, а также Э. Жису, 
А. Б. Катку, М. Шубу и А. И. Буфетову за плодотворное обсуждение и интерес к работе.

Авторам известно, что результат, аналогичный основным результатам настоящей работы, был получен Г. Фюрстенбергом, но не был им опубликован.

Кроме того, авторы чрезвычайно признательны рецензенту за проделанный им труд и за предоставленные ссылки.

1.3. Обозначения и определения. Опишем стандартную конструкцию случайной динамической системы с конечным числом отображений и независимыми итерациями (в настоящей работе мы рассматриваем только такие случайные динамические системы, поэтому далее слова «с конечным числом отображений и независимыми итерациями» мы будем опускать). Пусть заданы $T_{1}, \ldots, T_{s}-$ непрерывные отображения метрического компакта $X$ в себя - и некоторые положительные числа $p_{1}, \ldots, p_{s}$, в сумме дающие $1,-$ вероятности их применения.

Обозначим через $\Sigma_{s}^{+}=\{1, \ldots, s\}^{\mathbb{N}}$ пространство односторонних последовательностей элементов множества $\{1, \ldots, s\}$, и пусть $\nu$ - мера Бернулли на нем, соответствующая данному распределению вероятностей: $\nu=\nu_{0}^{\mathbb{N}}$, где $\nu_{0}(\{i\})=$ $p_{i}, i=1, \ldots, s$.

Мы будем различать понятия «динамическая система с несколькими отображениями» и «случайная динамическая система»:

ОПРеДЕЛЕНИЕ 1 . Динамической системой $\left(X ; T_{1}, \ldots, T_{s}\right)$ назовем гомоморфизм $\psi$ из свободной полугруппы $F_{s}=\left\langle t_{1}, \ldots, t_{s}\right\rangle$ в полугруппу непрерывных отображений компакта $X$ в себя, заданный соотношениями $t_{j} \mapsto T_{j}, j=1, \ldots, s$.

ОПРЕДЕЛЕНИЕ 2. Последовательность итерачий динамической системы $\left(X ; T_{1}, \ldots, T_{s}\right)$, соответствующая полубесконечному слову $\omega=\omega_{1} \omega_{2} \cdots \in \Sigma_{s}^{+},-$ это последовательность $\{T(n, \omega)\}_{n=0}^{\infty}$ непрерывных отображений из $X$ в себя, заданная правилом

$$
T(n, \omega)=T_{\omega_{n}} \circ T(n-1, \omega), \quad n \in \mathbb{N}, \quad T(0, \omega)=\mathrm{id} .
$$

Замечание 1. $T(n, \omega)=T_{\omega_{n}} \circ \cdots \circ T_{\omega_{2}} \circ T_{\omega_{1}}=\psi\left(t_{\omega_{n}} \cdots t_{\omega_{1}}\right), n \in \mathbb{N} \cup\{0\}$.

ОПРЕДЕЛЕНИЕ 3. Последовательность итераций случайной динамической системь $\left(X ; T_{1}, \ldots, T_{s} ; p_{1}, \ldots, p_{s}\right)$ - случайная последовательность $\{T(n, \omega)\}_{n=0}^{\infty}$ непрерывных отображений из $X$ в себя. Здесь $\omega \in \Sigma_{s}^{+}$выбирается случайно в соответствии с мерой $\nu$, заданной вероятностями $p_{i}$.

ЗАмЕчАниЕ 2. В результате мы имеем последовательность отображений, в которой каждое следующее получается из предыдущего применением слева одного из $T_{i}$. В силу того, что $\nu$ - мера Бернулли, выбор $T_{i}$ на разных этапах независим, $T_{j}$ выбирается с вероятностью $p_{j}$.

Как видим, случайная динамическая система отличается от динамической системы с несколькими отображениями тем, что в ней заданы вероятности применения отображений $T_{i}$, что позволяет говорить о последовательности итераций как об одной (хоть и случайной) последовательности.

Мы будем различать свойства, относящиеся именно к случайной динамической системе (утверждения вида сходимости почти наверное и т. д.) и к соответствующей динамической системе с несколькими отображениями (минимальность и т.д.). Кроме того, некоторые утверждения оказывается удобным формулировать с использованием еще одного понятия - полугруппы, порожденной отображениями $T_{1}, \ldots, T_{s}$. Мы обозначим эту полугруппу через $G^{+}=$ 
$G^{+}\left(T_{1}, \ldots, T_{s}\right)$. Как легко видеть, $G^{+}$- образ гомоморфизма $\psi$ из определения 1.

ОПРЕДЕЛЕНИЕ 4. Система $\left(X ; T_{1}, \ldots, T_{s}\right)$ называется минималъной, если любое замкнутое подмножество $M \subset X$, такое, что $T_{i}(M) \subset M$ при всех $i$, пусто или совпадает с $X$. В этом случае мы также будем говорить, что система $\left(T_{1}, \ldots, T_{s}\right)$ минимальна на $X$.

ЗАмЕчАНИЕ 3. Определение 4 можно переформулировать следующим образом: все $G^{+}$-орбиты плотны.

Теперь даны все определения, используемые в формулировке основного результата статьи: теоремы 1 разд. 2.1, дающей достаточные условия сближения орбит случайной динамической системы на окружности. Для формулировки остальных результатов понадобятся дальнейшие определения.

Оказывается, удобно изменить порядок применения отображений при итерировании: рассмотреть новую последовательность итераций, в которой на каждом шаге применяется то же отображение, что и раньше, но не слева, а справа. Фигурально говоря, если при обычном итерировании происходит «движение в будущее», то при таком - «уточнение прошлого»:

ОПРЕДЕЛЕНИЕ 5. Последовательность $T[n, \omega]$ итеращий с обращенным порядком системы $\left(X ; T_{1}, \ldots, T_{s}\right)$, соответствующая слову $\omega \in \Sigma_{s}^{+}$, - это последовательность непрерывных отображений компакта $X$ в себя, построенная по правилу

$$
T[n, \omega]=T[n-1, \omega] \circ T_{\omega_{n}}, \quad T[0, \omega]=\mathrm{id} .
$$

ЗАмЕчаниЕ 4. $T[n, \omega]=T_{\omega_{1}} \circ \cdots \circ T_{\omega_{n}}=\psi\left(t_{\omega_{1}} \cdots t_{\omega_{n}}\right), n \in \mathbb{N} \cup\{0\}$.

Кроме того, положим

$$
T_{\omega}=\psi\left(t_{\omega_{1}} \cdots t_{\omega_{n}}\right)=T_{\omega_{1}} \circ \cdots \circ T_{\omega_{n}}
$$

для конечного слова $\omega=\omega_{1} \cdots \omega_{n}$ в алфавите $\{1, \ldots, s\}$.

Обозначим меру Лебега на окружности через $\mu_{L}$, а меру, сосредоточенную в точке $x$, через $\delta_{x}$ (такую меру будем называть $\delta$-мерой). Будем говорить, что точка $x$ - атом веса $q>0$ для меры $\mu$, если $\mu(\{x\})=q$.

Для произвольного метрического компакта $K$ через $\mathscr{M}(K)$ обозначим пространство вероятностных мер на $K$. Как известно, слабая сходимость на $\mathscr{M}(K)$ метризуема, причем по отношению к этой метрике множество $\mathscr{M}(K)$ само является компактом.

Если дано непрерывное отображение $T: K \rightarrow L$, то будем обозначать через $\mathbf{T}$ или через $T_{*}$ индуцированное им отображение из $\mathscr{M}(K)$ в $\mathscr{M}(L)$ :

$$
(\mathbf{T} \mu)(A)=\left(T_{*} \mu\right)(A)=\mu\left(T^{-1}(A)\right), \quad A \subset L .
$$

Кроме того, если $K, L$ - метрические компакты, то можно рассматривать $\mathbf{T}: \mathscr{M}(K) \rightarrow \mathscr{M}(L)$ как отображение метрических компактов. В этой ситуации поднятие отображения $\mathbf{T}$ до отображения мер на пространствах мер - из $\mathscr{M}(\mathscr{M}(K))$ в $\mathscr{M}(\mathscr{M}(L))$ - будем обозначать через $\mathbf{T}_{*} \cdot$

Все меры, о которых будет идти речь в настоящей статье, предполагаются вероятностными.

ОПРЕДЕЛЕНИЕ 6. Мера $\mu_{H}$ на $K$ называется стаиионарной или гармонической, для системы $\left(K ; T_{1}, \ldots, T_{s} ; p_{1}, \ldots, p_{s}\right)$, если $\sum_{i=1}^{s} p_{i} \mathbf{T}_{i} \mu_{H}=\mu_{H}$. В этом случае мы также будем говорить, что мера $\mu_{H}$ стационарна для отображений $T_{i}$, взятых с весами $p_{i}$. 
Понятие стационарной меры для случайных динамических систем является обобщением понятия инвариантной меры для классических систем. Как и инвариантная мера в случае одного отображения, на компакте для случайной динамической системы стационарная мера всегда существует и может быть получена применением аналога процедуры Крылова-Боголюбова (см. [8, Lemma 2.2.1]).

ОПРеДЕЛЕНИЕ 7. Разбросом для меры $\mu$ на окружности назовем величину

$$
D(\mu)=\inf \left\{\varepsilon>0\left|\exists I \subset S^{1},\right| I \mid<\varepsilon: \mu(I)>1-\varepsilon\right\},
$$

где $I$ - дуга, а $|I|-$ ее длина.

ЗАмечание 5. $D(\mu)=0$ тогда и только тогда, когда $\mu$ есть $\delta$-мера.

Достаточно часто по полубесконечному слову $\omega$ мы будем строить какой-либо (вполне определенный) элемент $\xi$ какого-либо множества $X$, задавая тем самым отображение $\xi: \Sigma_{s}^{+} \rightarrow X$. В этом случае распределением значений $\xi$ мы будем называть распределение значений $\xi$ как случайной величины на вероятностном пространстве $\left(\Sigma_{s}^{+}, \nu\right)$, т. е. меру на $X$, полученную как $\xi_{*} \nu$.

\section{§2. Основные результаты}

Здесь и далее $T_{1}, \ldots, T_{s}$ - гомеоморфизмы окружности, сохраняющие ориентацию.

\section{1. Формулировки.}

ТеОрема 1. Пусть выполнены следующие предположения:

1) в полугруппе $G^{+}$найдется отображение $T_{\alpha}=T_{\alpha_{1}} \circ \cdots \circ T_{\alpha_{m}}$, являющееся гомеоморфизмом с ровно двумя неподвижными точками, одной притягивающей и одной отталкивающей;

2) система $\left(S^{1} ; T_{1}, \ldots, T_{s}\right)$ является минимальной;

3) система $\left(S^{1} ; T_{1}^{-1}, \ldots, T_{s}^{-1}\right)$, порожденная обратными отображениями, также минимальна.

Тогда для любого распределения положительных вероятностей $p_{i}$ почти наверное по (соответствующей ему) мере $\nu$ существует такое $z=z(\omega)$, что для любых $x, y \neq z$

$$
\lim _{n \rightarrow \infty} \rho(T(n, \omega)(x), T(n, \omega)(y))=0,
$$

где $\rho$ - метрика на окружности. Кроме того,

$$
\forall z_{0} \in S^{1} \quad \nu\left\{\omega \mid z(\omega)=z_{0}\right\}=0 .
$$

СледСтвиЕ 1. В предположениях теоремы 1 для любых $x, y \in S^{1}$ почти наверное по мере $\nu$

$$
\lim _{n \rightarrow \infty} \rho(T(n, \omega)(x), T(n, \omega)(y))=0 .
$$

Оказывается, что эта теорема является «наблюдаемым проявлением» другой теоремы, которая, как нам кажется, является более естественной. В ней порядок итераций заменяется на обратный, при этом последовательность итераций стационарной меры становится мартингалом (со значениями в пространстве мер на окружности), и, именно исходя из мартингальных свойств, мы доказываем эту новую теорему. Все остальные теоремы выводятся из нее применением несложных технических утверждений. 
Отметим, что, в отличие от обычных динамических систем, для которых минимальность отображения $T: X \rightarrow X$ (т. е. минимальность системы $(X ; T))$ эквивалентна минимальности отображения $T^{-1}$, в нашем случае может быть так, что одна из систем $\left(S^{1} ; T_{1}, \ldots, T_{s}\right)$ и $\left(S^{1} ; T_{1}^{-1}, \ldots, T_{s}^{-1}\right)$ минимальна, а другая - нет. Впрочем, имеющийся пример весьма техничен и потому здесь не приводится.

ТЕОРема 2. Пусть выполнены предположсения теоремы 1. Пусть мера $\mu_{H}$ является стационарной для системы $\left(S^{1} ; T_{1}, \ldots, T_{s} ; p_{1}, \ldots, p_{s}\right)$. Тогда

1) при $\nu$-почти всех $\omega$ существует предел

$$
\lim _{n \rightarrow \infty} \mathbf{T}[n, \omega]\left(\mu_{H}\right)=\lim _{n \rightarrow \infty}\left(\mathbf{T}_{\omega_{1}} \circ \cdots \circ \mathbf{T}_{\omega_{n}}\right)\left(\mu_{H}\right)=\mathscr{L}(\omega)
$$

2) предельная мера $\nu$-почти наверное является $\delta$-мерой: существует такое измеримое отображение $f^{+}: \Sigma_{s}^{+} \rightarrow S^{1}$, что $\nu$-почти наверное $\mathscr{L}(\omega)$ $=\delta_{f+(\omega)} \cdot$

Первое из утверждений этой теоремы - существование предела - хорошо известный даже в более общем случае факт, см. ссылки на литературу к теореме 6 §. Атомарность такого предела в контексте случайных блужданий применяется для описания их границ Пуассона (см. [8]), однако в контексте случайных динамических систем на окружности этот факт ранее известен не был.

СлЕДСТВИЕ 2. В предположениях теоремы 1 для любого набора вероятностей $p_{i}>0$ стационарная мера для системь $\left(S^{1} ; T_{1}, \ldots, T_{s} ; p_{1}, \ldots, p_{s}\right)$ единственна (хотя меры, соответствующие разным наборам вероятностей, могут различаться).

Еще одним следствием теоремы 2 является такая теорема, также описывающая наблюдаемую в компьютерных экспериментах динамику:

ТеОремА 3. Пусть $\mu$ - произвольная мера на окружности, не имеющая атомов. Тогда в предположениях теоремы 1 почти наверное (по мере Бернуллu $\nu)$

$$
\lim _{n \rightarrow \infty} D(\mathbf{T}(n, \omega)(\mu))=0 .
$$

В частности, в качестве $\mu$ можно взять меру Лебега $\mu_{L}$.

2.2. Следствия для косых произведений. Пусть для системы $\left(S^{1} ; T_{1}\right.$, $\left.\ldots, T_{s}\right)$ выполнены условия теоремы 1. Рассмотрим соответствующее этой системе косое произведение

$$
F: \Sigma_{s} \times S^{1} \rightarrow \Sigma_{s} \times S^{1}, \quad F(\omega, x)=\left(\sigma \omega, T_{\omega_{0}}(x)\right),
$$

над пространством двусторонних последовательностей $\Sigma_{s}=\{1, \ldots, s\} \mathbb{Z}$. Здесь $\sigma$ - сдвиг Бернулли на $\Sigma_{s}:(\sigma \omega)_{k}=\omega_{k+1}$.

Рассмотрим естественную меру на $\Sigma_{s} \times S^{1}-$ произведение меры Бернулли $\widetilde{\nu}=\nu_{0}^{\mathbb{Z}}$ в базе $\Sigma_{s}$ и меры Лебега $\mu_{L}$ в слое $S^{1}$. Возникает естественный вопрос: как ведут себя образы этой меры под действием $\mathbf{F}^{n}$ при $n \rightarrow \pm \infty$ ?

Ответом на этот вопрос является теорема 4, несложно выводящаяся из обобщений теоремы 2 - теоремы 7 и замечания 9 (см. с. 49).

Пусть $f^{+}$- отображение из теоремы 2, a $f^{-}$- отображение из теоремы 2 для системы $\left(S^{1} ; T_{1}^{-1}, \ldots, T_{s}^{-1}\right)$. Рассмотрим сечения $\varphi^{+}(x), \varphi^{-}(x): \Sigma_{s} \rightarrow \Sigma_{s} \times S^{1}$, определенные формулами

$$
\varphi^{+}(\omega)=\left(\omega, f^{+}\left(\omega_{-1} \omega_{-2} \ldots\right)\right), \quad \varphi^{-}(\omega)=\left(\omega, f^{-}\left(\omega_{0} \omega_{1} \omega_{2} \ldots\right)\right) .
$$


Теорема 4. Пусть $\mu$ - мера на окружности, не имеющая атомов, носителем которой является вся окружность (в частности, можно взять в качестве $\mu$ меру Лебега). Пусть $\lambda=\widetilde{\nu} \times \mu-$ мерана $\Sigma_{s} \times S^{1}$. Тогда

$$
\lim _{n \rightarrow+\infty} \mathbf{F}^{n} \lambda=\varphi^{+} \nu, \quad \lim _{n \rightarrow-\infty} \mathbf{F}^{n} \lambda=\varphi^{-} \nu
$$

Иными словами, в косом произведении есть два измеримых инвариантных сечения, первое из которых разумно считать аттрактором, а второе - репеллером, так как при итерациях естественной меры $\lambda$ она стремится к мере, сосредоточенной на этих сечениях, в прямом и обратном времени соответственно. Более того, эти сечения независимы (как случайные величины на базе со значениями в слое) - одно из них зависит только от «будущего», а другое только от «прошлого»! Впрочем, оба эти сечения оказываются всюду плотными, поэтому они не являются аттрактором и репеллером в классическом смысле этого слова.

2.3. Обобщения основных результатов. Формулируемая (без доказательства) в этом разделе теорема позволяет в случае гладких $T_{i}$ ослабить предположение 1) теоремы 1 . Ее доказательство будет приведено в отдельной работе.

Обозначим через $M S=M S\left(T_{1}, \ldots, T_{s}\right)$ подмножество в $G^{+}\left(T_{1}, \ldots, T_{s}\right)$, состоящее из отображений типа Морса-Смейла. Для любого $T \in M S$ обозначим через $A(T)$ число его притягивающих периодических точек (точки одной орбиты считаются различными). Пусть

$$
l\left(T_{1}, \ldots, T_{s}\right)=\min _{T \in M S\left(T_{1}, \ldots, T_{s}\right)} A(T) .
$$

ОПРеДЕЛЕНИЕ 8. Будем говорить, что система $\left(S^{1} ; T_{1}, \ldots, T_{s}\right)$ допускает $l$-листную факторизацию $(l>1)$, если существует $l$-листное (топологическое) накрытие $\pi: S^{1} \rightarrow S^{1}$, такое, что отображения системы «проносятся сквозь $\pi$ »: найдутся гомеоморфизмы окружности $\theta_{i}$, удовлетворяющие условию $\theta_{i} \circ \pi=$ $\pi \circ T_{i}$. Систему $\left(S^{1} ; \theta_{1}, \ldots, \theta_{s}\right)$ будем называть факторизованной системой.

Теорема 5. Пусть для системы $\left(S^{1} ; T_{1}, \ldots, T_{s}\right)$ выполнены предположения 2) и 3) теоремы 1 и, кроме того, найдется композиция отображсений $T_{i}$, являющаяся отображением типа Морса-Смейла. Пусть $l=l\left(T_{1}, \ldots, T_{s}\right)$. Тогда либо $l=1$ и, следовательно, мы находимся в условиях применимости теоремы 1, либо система допускает l-листную факторизачию.

В последнем случае $(l>1)$, как легко видеть, отображение типа МорсаСмейла с $l$ притягивающими (и, следовательно, с $l$ отталкивающими) периодическими точками после факторизации превращается в отображение с одной притягивающей и одной отталкивающей неподвижными точками, и, значит, мы опять находимся в условиях применимости теоремы 1. (Минимальность системы сохраняется при факторизации.)

Пример двусторонне минимальной системы, содержащей хотя бы одно отображение типа Морса-Смейла и не имеющей композиции с одной отталкивающей и одной притягивающей неподвижными точками, легко получить, обратив процедуру факторизации - подняв отображения на накрытие. Действительно, возьмем любую гладкую систему $\left(\theta_{1}, \ldots, \theta_{s}\right)$, удовлетворяющую условиям теоремы 1 , и пусть $\pi(x)=l x$. Тогда, как легко видеть, при каждом $i$ композиция $\left(\pi^{-1} \circ \theta_{i} \circ \pi\right)(x)=\frac{1}{l} \theta_{i}(l x)-$ многозначная функция на окружности с тривиальной монодромией, а значит, существует ее однозначная ветвь $T_{i}$. Ясно, что получающаяся система $\left(S^{1} ; T_{1}, \ldots, T_{s}\right)$ не содержит в своей полугруппе 
$G^{+}\left(T_{1}, \ldots, T_{s}\right)$ отображений с одной отталкивающей и одной притягивающей неподвижными точками. Теорема 5 утверждает, что факторизуемые системы единственный класс таких примеров.

СлЕДСТВИЕ 3. Если система $\left(S^{1} ; T_{1}, \ldots, T_{s}\right)$ удовлетворяет предположениям 2) и 3) теоремы 1 и не допускает факторизации, то она удовлетворяет и предположению 1), а следовательно, для нее выполняются заключения теорем 1 u 2.

2.4. Схема доказательства основных результатов. В $§ 3$ будет показано, что предел в теореме 2 существует, хотя, возможно, и не является атомарной мерой. Этот предел - случайная величина, и можно рассмотреть распределение его значений, являющееся мерой на пространстве мер на $S^{1}$. Оказывается, что это распределение значений стационарно относительно $\mathbf{T}_{i}$, взятых с теми же весами $p_{i}$. В $\S 4$ будет показано, что в предположениях теоремы 1 всякая мера на пространстве мер, стационарная относительно $\mathbf{T}_{i}$, сосредоточена на множестве $\delta$-мер. В 55 из этих двух фактов будет выведена теорема 2 , а затем из нее будут выведены все остальные теоремы.

\section{§3. Сходимость при обращенном порядке итераций и свойства предела}

Нам понадобится следующая теорема, принадлежащая Г. Фюрстенбергу, которая выводится из теоремы о сходимости мартингалов. Эта теорема была опубликована в более общем виде в его работе [5, Theorem 7.5]; ее изложение также можно найти в работах [8, Lemma 2.2.3] и [3, Lemma 1.1].

Теорема 6. Пусть $X-$ компакт, $T_{1}, \ldots, T_{s}$ - его непрерывные отображения в себя и $\mu_{H}$ - стационарная относительно этих отображсний мера. Тогда существует измеримое (в смысле борелевских б-алгебр метрических компактов) отображсение $\mathscr{L}$ пространства $\Sigma_{s}^{+}$в пространство вероятностных мер на $X$, такое, что

$$
(\mathbf{T}[n, \omega]) \mu_{H} \stackrel{\nu-n . \mu .}{\longrightarrow} \mathscr{L}(\omega) \quad(n \rightarrow \infty) .
$$

Из этой теоремы следует первое из двух утверждений теоремы 2 - существование, хотя, быть может, и не атомарного, предела.

ПРЕДЛОЖЕНИЕ 1. В условияХ теоремъ 6 образ меръ Бернулли $\nu$ под действием $\mathscr{L}_{*}$ - мера, стационарная для системы $\left(\mathscr{M}\left(S^{1}\right) ; \mathbf{T}_{1}, \ldots, \mathbf{T}_{s} ; p_{1}, \ldots, p_{s}\right)$.

ДокАЗАтЕльство. Для каждого $j \in\{1, \ldots, s\}$ следующая диаграмма коммутативна:

Действительно,

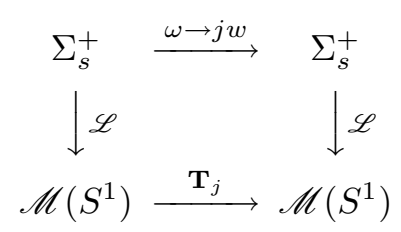

$\mathbf{T}_{j} \mathscr{L}(\omega)=\mathbf{T}_{j} \lim _{n \rightarrow \infty}\left(\mathbf{T}_{\omega_{1}} \circ \cdots \circ \mathbf{T}_{\omega_{n}}\right)\left(\mu_{H}\right)=\lim _{n \rightarrow \infty}\left(\mathbf{T}_{j} \circ \mathbf{T}_{\omega_{1}} \circ \cdots \circ \mathbf{T}_{\omega_{n}}\right)\left(\mu_{H}\right)=\mathscr{L}(j \omega)$.

Мера Бернулли $\nu$ стационарна относительно отображений $\omega \rightarrow j w$, взятых с весами $p_{j}$; следовательно, ее образ под действием $\mathscr{L}$ стационарен относительно отображений $\mathbf{T}_{j}$, взятых с теми же весами. 


\section{§4. Атомарность предельной меры}

Пусть $\Delta-$ подмножество в $\mathscr{M}\left(S^{1}\right)$, состоящее из $\delta$-мер. Докажем следующую лемму:

Лемма 1. В предположениях теоремы 1 любая мера $M$ на $\mathscr{M}\left(S^{1}\right)$, стационарная относительно отображсений $\mathbf{T}_{i}$, сосредоточена на $\Delta$.

Для доказательства леммы 1 нам понадобятся следующие два определения. Они общеизвестны и в более общем виде могут быть найдены, например, в книге Ширяева [2], но для полноты изложения мы приводим их здесь (в частном случае дискретного времени $t \in \mathbb{N} \cup\{0\})$.

Пусть на вероятностном пространстве $(\Omega, \mathscr{F}, P)$ задана возрастающая последовательность $\sigma$-алгебр $\left\{\mathscr{F}_{t}\right\}_{t \in \mathbb{N} \cup\{0\}}$, каждая из которых есть подалгебра $\sigma$-алгебры $\mathscr{F}$,

$$
\mathscr{F}_{0} \subset \mathscr{F}_{1} \subset \cdots \subset \mathscr{F} .
$$

ОПРЕДЕЛЕНИЕ 9. Случайный процесс $\chi_{t}(\omega)$, измеримый относительно возрастающей последовательности $\sigma$-алгебр $\mathscr{F}_{t}$ (при всех $t$ случайная величина $\chi_{t}$ измерима относительно $\left.\mathscr{F}_{t}\right)$, называется мартингалом, если $E\left(\chi_{t} \mid \mathscr{F}_{s}\right)=\chi_{s}$ для любых $s, t \in \mathbb{N} \cup\{0\}, s<t$.

ОПредЕлЕниЕ 10. Случайный момент времени $\tau: \Omega \rightarrow(\mathbb{N} \cup\{0\}) \cup\{\infty\}$ называется марковским, если для любого момента времени $t \in \mathbb{N} \cup\{0\}$ событие $\{\omega \mid \tau(\omega) \leqslant t\}$ принадлежит $\sigma$-алгебре $\mathscr{F}_{t}$.

ДокАзАТЕльство лЕммы 1. Для доказательства этого утверждения возьмем разброс меры $D(\mu)$ и проинтегрируем его по всему пространству мер по мере $M$. Поскольку разброс неотрицателен и равен 0 на $\delta$-мерах и только на них, то достаточно показать, что этот интеграл равен 0 .

Для этого рассмотрим целое семейство подобных интегралов - случайный процесс с дискретным временем

$$
\chi(n, \omega)=\chi_{n}\left(\omega_{1}, \ldots, \omega_{n}\right)=\int_{\mathscr{M}\left(S^{1}\right)} D \circ \mathbf{T}[n, \omega] d M .
$$

Заметим, что это - мартингал (относительно последовательности цилиндрических $\sigma$-алгебр). Для этого достаточно проверить, что

$$
\chi_{n}\left(\omega_{1}, \ldots, \omega_{n}\right)=\sum_{j=1}^{s} p_{j} \chi_{n+1}\left(\omega_{1}, \ldots, \omega_{n}, j\right) .
$$

Действительно,

$$
\begin{aligned}
\sum_{j=1}^{s} p_{j} \chi_{n+1}\left(\omega_{1}, \ldots, \omega_{n}, j\right)=\sum_{j=1}^{s} p_{j} \int_{\mathscr{M}\left(S^{1}\right)} D \circ \mathbf{T}_{[\omega]_{n}} \circ \mathbf{T}_{j} d M \\
\quad=\sum_{j=1}^{s} p_{j} \int_{\mathscr{M}\left(S^{1}\right)} D \circ \mathbf{T}[n, \omega] d\left(\left(\mathbf{T}_{j}\right)_{*} M\right)=\int_{\mathscr{M}\left(S^{1}\right)} D \circ \mathbf{T}[n, \omega] d\left(\sum_{j=1}^{s} p_{j}\left(\mathbf{T}_{j}\right)_{*} M\right) \\
=\int_{\mathscr{M}\left(S^{1}\right)} D \circ \mathbf{T}[n, \omega] d M=\chi_{n}\left(\omega_{1}, \ldots, \omega_{n}\right)
\end{aligned}
$$

(равенство $\sum_{j=1}^{s} p_{j}\left(\mathbf{T}_{j}\right)_{*} M=M$ выполнено в силу стационарности меры $\left.M\right)$. 
Заметим, что процесс $\chi$ ограничен (равномерно по $n$ ) и неотрицателен:

$$
\chi(n, \omega)=\chi_{n}\left(\omega_{1}, \ldots, \omega_{n}\right)=\int_{\mathscr{M}\left(S^{1}\right)} D \circ \mathbf{T}[n, \omega] d M \leqslant \sup _{\mu \in \mathscr{M}\left(S^{1}\right)} D(\mu) .
$$

Зафиксируем произвольное $\varepsilon>0$ и рассмотрим марковский момент

$$
k_{\varepsilon}(\omega)=\min \{n \geqslant 0 \mid \chi(n, \omega)<\varepsilon\} .
$$

(Минимум пустого множества полагаем равным $\infty$; момент $k_{\varepsilon}(\omega)$ марковский, так как наступление события

$$
k_{\varepsilon}(\omega) \leqslant n \Longleftrightarrow \exists n_{1} \leqslant n: \chi\left(n_{1}, \omega\right)<\varepsilon
$$

может быть определено лишь по первым $n$ координатам слова $\omega$, а следовательно, это событие принадлежит цилиндрической $\sigma$-алгебре первых $n$ координат.) Докажем, что момент $k_{\varepsilon}(\omega)$ конечен почти наверное.

ЛЕмма 2. В условиях теоремы 1 для каждого $\varepsilon>0$ момент $k_{\varepsilon}(\omega)$ конечен ע-почти наверное.

ЗАмЕчАнИЕ 6. Именно эта лемма существенно использует тот факт, что фазовым пространством является окружность. В случае когда размерность фазового пространства больше 1, может существовать сколь угодно много попарно непересекающихся множеств большого диаметра - ситуация, невозможная на окружности. Из-за этого перестает быть верной первая часть предложения 3 , использующая совпадение понятий «диаметр» и «лебегова мера» для связных множеств в одномерной ситуации.

ДокАЗАтЕЛьство леммы 2. Достаточно доказать, что

$$
\forall \varepsilon>0 \exists l: \forall N, \forall \omega_{1} \cdots \omega_{N} \exists l^{\prime} \leqslant l, \exists \omega_{N+1} \cdots \omega_{N+l^{\prime}}: \chi_{N+l^{\prime}}\left(\omega_{1} \cdots \omega_{N+l^{\prime}}\right)<\varepsilon .
$$

Действительно, отсюда следует, что

$$
\nu\left\{k_{\varepsilon}(\omega)>N+l\right\} \leqslant\left(1-\min \left(p_{j}\right)^{l}\right) \cdot \nu\left\{k_{\varepsilon}(\omega)>N\right\} .
$$

Поэтому $\nu\left\{k_{\varepsilon}(\omega)>N\right\} \leqslant\left(1-\min \left(p_{j}\right)^{l}\right)^{[N / l]} \rightarrow 0$ при $N \rightarrow \infty$, т. е. момент $k_{\varepsilon}$ конечен $\nu$-почти наверное.

Докажем, что имеет место утверждение (1). А именно, пусть задан гомеоморфизм окружности $T_{\omega_{1} \cdots \omega_{N}}=g$. Предъявим такие $\omega_{N+1}, \ldots, \omega_{N+l^{\prime}}$, что

$$
\int_{\mathscr{M}\left(S^{1}\right)} D \circ \mathbf{g} \circ \mathbf{T}_{\omega_{N+1} \ldots \omega_{N+l^{\prime}}} d M<\varepsilon,
$$

причем $l^{\prime}$ не превосходит некоторой константы, не зависящей от $g$. Для этого достаточно построить такие $\omega_{N+1}, \ldots, \omega_{N+l^{\prime}}$, что

$$
\exists I \subset S^{1},|I|<\varepsilon: \quad M\left\{\mu \mid\left(\left(\mathbf{g} \circ \mathbf{T}_{\omega_{N+1} \ldots \omega_{N+l^{\prime}}}\right) \mu\right)(I)>1-\varepsilon\right\}>1-\varepsilon
$$

(правда, отсюда следует лишь неравенство $\chi_{N+l^{\prime}}\left(\omega_{1} \cdots \omega_{N+l^{\prime}}\right)<\left(1+\max _{\mathscr{M}\left(S^{1}\right)} D\right) \varepsilon$, но в силу произвольности $\varepsilon$ достаточно доказать (2)).

ПРЕДЛОЖЕНИЕ 2. В предположениях теоремы 1 орбита любой точки а под действием полугруппь $G^{+}\left(T_{1}, \ldots, T_{s}\right)$ бесконечна.

ДокАзАтЕльство. Если орбита конечна, то она является замкнутым (в силу конечности) инвариантным множеством, отличным от пустого и всей окружности, что противоречит минимальности системы. 
СлЕДСТВИЕ 4. При тех же предположениях орбита любой точки $r$ под действием $G^{+}\left(T_{1}^{-1}, \ldots, T_{s}^{-1}\right)$ бесконечна.

Пусть $T_{\alpha}$ - гомеоморфизм из условия 1$)$ теоремы $1, a$ - его притягивающая неподвижная точка, $r$ - отталкивающая. Выберем $p>1 / \varepsilon$ образов точки $a$ под действием $G^{+}\left(T_{1}, \ldots, T_{s}\right)$ и обозначим их через $a_{j}$, выберем $q>1 / \varepsilon^{2}$ образов точки $r$ под действием $G^{+}\left(T_{1}^{-1}, \ldots, T_{s}^{-1}\right)$ и обозначим их через $r_{j}$. Выберем попарно непересекающиеся окрестности $U_{j}$ точек $a_{j}$ и попарно непересекающиеся окрестности $V_{j}$ точек $r_{j}$.

ПредЛОЖенИЕ 3. 1. Найдется $j_{1}$, такое, что образ окрестности $U_{j_{1}}$ под действием $g$ имеет длину, менъшую $\varepsilon$.

2. Найдется $j_{2}$, такое, что $M\left\{\mu \mid \mu\left(V_{j_{2}}\right) \geqslant \varepsilon\right\}<\varepsilon$.

ДокАзАТЕЛЬСтво. 1. Поскольку $g$ - гомеоморфизм, образы непересекающихся дуг - непересекающиеся дуги. Если длины образов всех $U_{j}$ не меньше $\varepsilon$, то их суммарная длина не меньше $p \varepsilon>1$, т. е. больше, чем длина окружности.

2. Пусть такого $j_{2}$ не найдется. Отметим, что для каждой меры $\mu \in \mathscr{M}\left(S^{1}\right)$ выполнено соотношение $\sum_{j=1}^{q} \mu\left(V_{j}\right)=\mu\left(\bigcup_{j=1}^{q} V_{j}\right) \leqslant 1$. Отсюда следует, что

$$
\int_{\mathscr{M}\left(S^{1}\right)} \sum_{j=1}^{q} \mu\left(V_{j}\right) d M \leqslant 1 .
$$

Тогда в силу неравенства Чебышёва

$$
\begin{aligned}
\int_{\mathscr{M}\left(S^{1}\right)} \sum_{j=1}^{q} \mu\left(V_{j}\right) d M & =\sum_{j=1}^{q} \int_{\mathscr{M}\left(S^{1}\right)} \mu\left(V_{j}\right) d M \\
& \geqslant \sum_{j=1}^{q} \varepsilon M\left\{\mu \mid \mu\left(V_{j}\right) \geqslant \varepsilon\right\} \geqslant \sum_{j=1}^{q} \varepsilon^{2}=q \varepsilon^{2}>1 .
\end{aligned}
$$

Противоречие.

Поскольку все точки $r_{j}$ были образами репеллера $r$ под действием отображений из полугруппы $G^{+}\left(T_{1}^{-1}, \ldots, T_{s}^{-1}\right)$, найдутся слова $v^{j}$, такие, что $T_{v^{j}}\left(r_{j}\right)=r$. Аналогично найдутся такие слова $u^{j}$, что $T_{u^{j}}(a)=a_{j}$. Кроме того, $T_{v^{i}}\left(V_{i}\right)-$ окрестность точки $r$, а $\left(T_{u^{j}}\right)^{-1}\left(U_{j}\right)$ - окрестность точки $a$; поэтому найдется такое $n_{i j} \in \mathbb{N}$, что

$$
T_{\alpha}^{n_{i j}}\left(S^{1} \backslash T_{v^{i}}\left(V_{i}\right)\right) \subset T_{u^{j}}^{-1}\left(U_{j}\right) .
$$

Положим $l=\max _{i, j}\left(\left|u^{j}\right|+n_{i j}|\alpha|+\left|v^{i}\right|\right)$, где $|u|-$ длина слова $u$.

В силу предложения 3 найдутся такие $j_{1}$ и $j_{2}$, что образ окрестности $U_{j_{1}}$ под действием гомеоморфизма $g$ имеет длину, меньшую $\varepsilon$, и $M\left\{\mu \mid \mu\left(V_{j_{2}}\right) \geqslant \varepsilon\right\}<\varepsilon$. Выберем в качестве слова $\omega_{N+1} \ldots \omega_{N+l^{\prime}}$ слово $u^{j_{1}} \omega^{j_{2} j_{1}} v^{j_{2}}$. Здесь слово $\omega^{j_{2} j_{1}}$ состоит из $n_{j_{2} j_{1}}$ слов $\alpha$, записанных подряд, $l^{\prime}=\left|u^{j_{1}}\right|+n_{j_{2} j_{1}}|\alpha|+\left|v^{j_{2}}\right|$. Покажем, что такой выбор удовлетворяет нашим требованиям.

Действительно, выберем в качестве $I$ дугу $g\left(U_{j_{1}}\right)$. В силу выбора $j_{1}$ выполнено неравенство $|I|<\varepsilon$. Заметим, что для большинства (в смысле меры $M$ ) мер $\mu \in \mathscr{M}\left(S^{1}\right)$ мера дуги $V_{j_{2}}$ мала, а именно, мера $M$ тех мер $\mu$, для которых $\mu\left(V_{j_{2}}\right)<\varepsilon$, больше $1-\varepsilon$.

Но под действием $T_{v^{j_{2}}}$ дуга $V_{j_{2}}$ переходит в окрестность точки $r$, причем под действием $T_{\omega^{j_{2} j_{1}}}=T_{\alpha}^{n_{j_{2} j_{1}}}$ дополнение до этой окрестности переходит внутрь 
$T_{u^{j_{1}}}^{-1}\left(U_{j_{1}}\right)-$ окрестности точки $a$ :

$$
T_{\alpha}^{n_{j_{2} j_{1}}}\left(S^{1} \backslash T_{v^{j_{2}}}\left(V_{j_{2}}\right)\right) \subset T_{u^{j_{1}}}^{-1}\left(U_{j_{1}}\right) .
$$

Отсюда в силу взаимной однозначности всех $T_{i}$ получаем

$$
T_{u^{j_{1}}}\left(T_{\alpha}^{n_{j_{2} j_{1}}}\left(T_{v^{j_{2}}}\left(S^{1} \backslash V_{j_{2}}\right)\right)\right) \subset U_{j_{1}},
$$

т. е. под действием $T_{\omega_{N+1} \ldots \omega_{N+l^{\prime}}}$ дополнение до $V_{j_{2}}$ переходит внутрь $U_{j_{1}}$. Тем самым для тех мер $\mu$, для которых $\mu\left(V_{j_{2}}\right)<\varepsilon$, выполняется неравенство

$$
\left(\mathbf{T}_{\omega_{N+1} \cdots \omega_{N+l^{\prime}}} \mu\right)\left(U_{j_{1}}\right)>1-\varepsilon,
$$

и, значит,

$$
\left(\left(\mathbf{g} \circ \mathbf{T}_{\omega_{N+1} \cdots \omega_{N+l^{\prime}}}\right) \mu\right)(I)>1-\varepsilon
$$

Отсюда

$$
M\left\{\mu \mid\left(\left(\mathbf{g} \circ \mathbf{T}_{\omega_{N+1} \cdots \omega_{N+l^{\prime}}}\right) \mu\right)(I)>1-\varepsilon\right\} \geqslant M\left\{\mu \mid \mu\left(V_{j_{2}}\right)<\varepsilon\right\}>1-\varepsilon .
$$

Слово $u^{j_{1}} \omega^{j_{2} j_{1}} v^{j_{2}}$ удовлетворяет условию леммы. Лемма 2 доказана.

ОКОНЧАНИЕ ДОКАЗАТЕЛЬСТВА ЛЕММЫ 1. ПоскольКу $\chi$ - ограниченный мартингал с дискретным временем, математическое ожидание его значения в конечный почти наверное марковский момент времени совпадает с его математическим ожиданием (см. [2, с. 477-478]). По лемме 2 момент $k_{\varepsilon}(\omega)$ конечен почти наверное. Получаем

$$
E \chi\left(k_{\varepsilon}(\omega), \omega\right)=E \chi(0, \omega)=\int_{\mathscr{M}\left(S^{1}\right)} D d M .
$$

С другой стороны, в силу определения $k_{\varepsilon}(\omega)$ левая часть может быть сделана сколь угодно малой:

$$
\chi\left(k_{\varepsilon}(\omega), \omega\right)<\varepsilon \Longrightarrow E \chi\left(k_{\varepsilon}(\omega), \omega\right)<\varepsilon .
$$

Отсюда $\int_{\mathscr{M}\left(S^{1}\right)} D d M=0$. Лемма 1 доказана.

\section{§5. Доказательство основных результатов}

\section{1. Доказательство теоремы 2.}

ДокАЗАТЕЛЬСтво ТЕОРЕМЫ 2. Поскольку распределение значений отображения $\mathscr{L}$ (мера на $\mathscr{M}\left(S^{1}\right)$, заданная как $\left.\mathscr{L}_{*} \nu\right)$ стационарно относительно отображений $\mathbf{T}_{i}$, утверждение теоремы получается как следствие леммы 1. При этом измеримость отображения $f^{+}$следует из измеримости $\mathscr{L}$.

Для стационарной меры $\mu$ обозначим через $f_{\mu}^{+}$функцию $f^{+}$из теоремы 2 , получающуюся при подстановке $\mu_{H}=\mu$.

ПреДЛОЖЕНИЕ 4. Распределение значений функиии $f_{\mu}^{+}, m . e . \mathbf{f}_{\mu}^{+} \nu$, совпадает с мерой $\mu$.

ДокАзАТЕЛьство. Известна следующая лемма (см. [5; 8, Lemma 2.2.3])

ЛЕмма 3. В условиях теоремы 6

$$
\mu_{H}=\int_{\Sigma_{s}} \mathscr{L}(\omega) d \nu(\omega) .
$$


ЗАмЕчАНИЕ 7. Эта лемма позволяет переставлять переход к пределу и математическое ожидание: в силу ее стационарности мера $\mu_{H}$ совпадает со средним своих итераций за любое фиксированное время $n$. Переходя к пределу под знаком интеграла, мы получили бы требуемое равенство. Лемма 3 утверждает, что такой переход законен.

Применение леммы 3 немедленно доказывает предложение 4:

$$
\mu=\int_{\Sigma_{s}^{+}} \mathscr{L}(\omega) d \nu(\omega)=\int_{\Sigma_{s}^{+}} \delta_{f_{\mu}^{+}(\omega)} d \nu(\omega)=\mathbf{f}_{\mu}^{+} \nu
$$

ДокАЗАТЕЛьСТво СЛЕДстВия 2. Пусть существуют две стационарных меры $\mu_{1}$ и $\mu_{2}$. Заметим, что полусумма этих стационарных мер также стационарна; поэтому $\nu$-почти наверное

$$
\left(\delta_{f_{\mu_{1}}^{+}(\omega)}+\delta_{f_{\mu_{2}}^{+}(\omega)}\right) / 2=\lim _{n \rightarrow \infty}(\mathbf{T}[n, \omega])\left(\left(\mu_{1}+\mu_{2}\right) / 2\right)=\delta_{f_{\left(\mu_{1}+\mu_{2}\right) / 2}^{+}(\omega)} .
$$

Полусумма $\delta$-мер является $\delta$-мерой, только если эти меры сосредоточены в одной и той же точке; поэтому $f_{\mu_{1}}^{+}(\omega)=f_{\mu_{2}}^{+}(\omega)$ почти наверное. Но в силу предложения 4 имеем $\mu_{1}=\mathbf{f}_{\mu_{1}}^{+} \nu=\mathbf{f}_{\mu_{2}}^{+} \nu=\mu_{2}$.

ЗАмЕчАниЕ 8. Проведенное доказательство аналогично доказательству в работе [8], см. теорему 2.2.4.

Для доказательства остальных теорем нам понадобится несколько технических утверждений, связанных со стационарной мерой.

\section{2. Свойства стационарной меры.}

ПРЕДЛОЖЕНИЕ 5. В предположениях теоремы 1 носитель стационарной меры - вся окружность.

ДокАЗАтельство. Обозначим носитель меры $\mu_{H}$ через $A$; тогда множество $A$ замкнуто и непусто. Кроме того, $\mu_{H}=\sum_{i=1}^{s} p_{i} \mathbf{T}_{i} \mu_{H}$. Поэтому

$$
A=\operatorname{supp}\left(\mu_{H}\right)=\bigcup_{i=1}^{s} \operatorname{supp}\left(\mathbf{T}_{i} \mu_{H}\right)=\bigcup_{i=1}^{s} T_{i}\left(\operatorname{supp}\left(\mu_{H}\right)\right)=\bigcup_{i=1}^{s} T_{i}(A),
$$

откуда следует, что $T_{i}(A) \subset A$ для любого $i$. В силу минимальности системы $A=S^{1}$.

ПРЕДЛОЖЕНИЕ 6. В предположениях теоремы 1 стаиионарная мера не содержит атомов, т. е. $\mu_{H}(\{b\})=0$ для любой точки b окружности.

ДокАЗАтельство. Пусть это не так. Назовем весом атома $b \in S^{1}$ число $\mu_{H}(\{b\})$. Тогда для любого $\varepsilon>0$ существует лишь конечное (не превосходящее $1 / \varepsilon)$ число атомов с весом, не меньшим $\varepsilon$. Поэтому существует наибольший вес $q$ и конечное множество $B$ атомов такого веса, $B=\left\{b \mid \mu_{H}(\{b\})=q\right\}$. Но тогда для любой точки $b \in B$

$$
q=\mu_{H}(\{b\})=\left(\sum_{i=1}^{s} p_{i} \mathbf{T}_{i} \mu_{H}\right)(\{b\})=\sum_{i=1}^{s} p_{i} \mu_{H}\left(\left\{T_{i}^{-1}(b)\right\}\right) \leqslant \sum_{i=1}^{s} p_{i} q=q,
$$

и равенство может достигаться, только если все $T_{i}^{-1}(b)$ также имеют вес $q$. Отсюда следует, что $B$ - конечное непустое инвариантное относительно всех отображений $T_{i}^{-1}$ множество, а это противоречит минимальности системы $\left(S^{1} ; T_{1}^{-1}, \ldots, T_{s}^{-1}\right)$. 
Известно следующее утверждение (мы приводим его здесь без доказательства):

ПРЕДЛОЖЕНИЕ 7. Пусть мера $\mu$ не имеет атомов, а ее носитель - вся окружность. Тогда $\mu$ гомеоморфна мере Лебега, m. е. $\mu=\mathbf{g} \mu_{L}$ для некоторого гомеоморбизма $g$.

Сопоставив это предложение с двумя предыдущими, получаем

СЛЕДСТВИЕ 5. В предположениях теоремы 1 стационарная мера гомеоморфна мере Лебега.

5.3. Эквивалентность условий сжатия меры в точку. Мы сформулируем без доказательства следующее техническое утверждение:

ПРЕДЛОЖЕНИЕ 8. Пусть $g_{1}, g_{2},\left\{R_{n}\right\}_{n \in \mathbb{N}}$ - гомеоморфизмы окружности, $b \in S^{1}$. Тогда следующие утверждения эквивалентны:

1) $\mathbf{R}_{n} \mu_{L} \rightarrow \delta_{b}$ npu $n \rightarrow \infty$;

2) $\mathbf{g}_{1} \mathbf{R}_{n} \mathbf{g}_{2} \mu_{L} \rightarrow \delta_{g_{1}(b)}$ npu $n \rightarrow \infty$

Эта эквивалентность легко выводится из равномерной непрерывности гомеоморфизмов $g_{i}$ и $g_{i}^{-1}$.

Из этого утверждения, следствия 5 и теоремы 2 вытекает

ТЕОРема 7. Пусть выполнены условия теоремы 1 u $f^{+}: \Sigma_{s}^{+} \rightarrow S^{1}$-отображсение из теоремы 2. Тогда

$$
\mathbf{T}[n, \omega] \mu_{L} \stackrel{\nu-n . \mu .}{\longrightarrow} \delta_{f+(\omega)} \quad(n \rightarrow \infty) .
$$

ДокАЗАтЕЛЬСтво. В силу следствия 5 имеем $\mu_{L}=\mathbf{g} \mu_{H}$ для некоторого гомеоморфизма окружности $g$. Тогда $\mathbf{T}[n, \omega] \mu_{L}=(\mathbf{T}[n, \omega] \circ \mathbf{g}) \mu_{H}$. В силу теоремы 2 имеем $\mathbf{T}[n, \omega] \mu_{H} \rightarrow \delta_{f+(\omega)}$ при $n \rightarrow \infty$. В силу предложения 8

$$
\mathbf{T}[n, \omega] \mu_{L}=(\mathbf{T}[n, \omega] \circ \mathbf{g}) \mu_{H} \rightarrow \delta_{f+(\omega)} \quad(n \rightarrow \infty) .
$$

ЗАмЕчАнИЕ 9. У тверждение теоремы 7 остается верным, если заменить меру Лебега $\mu_{L}$ на любую другую меру $\mu$ на окружности, не имеющую атомов.

ПРЕДЛОЖЕНИЕ 9. Если $h$ - гомеоморфизм, то $D\left(\mathbf{h} \mu_{L}\right)=D\left(\mathbf{h}^{-1} \mu_{L}\right)$.

ДокАЗАтельство. Действительно, если для некоторого $\varepsilon>0$ нашлась дуга $I$ длины, меньшей $\varepsilon$, такая, что $\left(\mathbf{h} \mu_{L}\right)(I)>1-\varepsilon$, то для дуги $I^{\prime}=S^{1} \backslash h(I)$ выполнены неравенства $\left|I^{\prime}\right|<\varepsilon$ и $\left(\mathbf{h}^{-1} \mu_{L}\right)\left(I^{\prime}\right)>1-\varepsilon$. Поэтому множества, инфимумы которых берутся в определении $D$, совпадают, а следовательно, совпадают и сами инфимумы.

ПРЕДЛОЖЕНИЕ 10. Пусть $\mu_{1}, \mu_{2}$ - две меръ на окружности. Тогда $D\left(\mu_{1}\right), D\left(\mu_{2}\right) \leqslant 2 D\left(\left(\mu_{1}+\mu_{2}\right) / 2\right)$.

ДокАЗАтЕЛьство. Пусть $D\left(\left(\mu_{1}+\mu_{2}\right) / 2\right)<\varepsilon$. Тогда найдется такая дуга $I$ длины, меньшей $\varepsilon$, что $\left(\left(\mu_{1}+\mu_{2}\right) / 2\right)(I)=\left(\mu_{1}(I)+\mu_{2}(I)\right) 2>1-\varepsilon$. Но в этом случае $\mu_{1}(I), \mu_{2}(I)>1-2 \varepsilon$. Следовательно, $D\left(\mu_{1}\right)<2 \varepsilon, D\left(\mu_{2}\right)<2 \varepsilon$. Переход к инфимуму завершает доказательство.

\section{4. Доказательство теорем 1 и 3.}

ДоКАЗАТЕЛЬСтво теОРЕмы 3. Рассмотрим для начала случай, когда носителем меры $\mu$ является вся окружность. Тогда в силу предложения 7 существует 
такой гомеоморфизм $g$, что $\mu=\mathbf{g} \mu_{L}$. Но

$$
\begin{aligned}
D\left(\left(\mathbf{T}_{\omega_{n}} \circ \cdots \circ \mathbf{T}_{\omega_{1}}\right) \mu\right)=D\left(\left(\mathbf{T}_{\omega_{n}} \circ \cdots \circ \mathbf{T}_{\omega_{1}} \circ \mathbf{g}\right) \mu_{L}\right) \\
\quad=D\left(\left(\left(\mathbf{T}_{\omega_{n}} \circ \cdots \circ \mathbf{T}_{\omega_{1}} \circ \mathbf{g}\right)^{-1}\right) \mu_{L}\right)=D\left(\left(\mathbf{g}^{-1} \circ \mathbf{T}_{\omega_{1}}^{-1} \circ \cdots \circ \mathbf{T}_{\omega_{n}}^{-1}\right) \mu_{L}\right)
\end{aligned}
$$

Заметим, что предположения теоремы 1 сохраняются при замене всех $T_{i}$ на $T_{i}^{-1}$. Поэтому в этих же предположениях в силу теоремы 7

$$
\left(\mathbf{T}_{\omega_{1}}^{-1} \circ \cdots \circ \mathbf{T}_{\omega_{n}}^{-1}\right) \mu_{L} \stackrel{\nu \text {-п.н. }}{\longrightarrow} \delta_{f^{+}(\omega)} \quad(n \rightarrow \infty) .
$$

Отсюда в силу предложения 8

$$
\left(\mathbf{g}^{-1} \circ \mathbf{T}_{\omega_{1}}^{-1} \circ \cdots \circ \mathbf{T}_{\omega_{n}}^{-1}\right) \mu_{L} \stackrel{\nu \text {-п.н. }}{\longrightarrow} \delta_{g^{-1}\left(f^{+}(\omega)\right)}(n \rightarrow \infty) .
$$

Значит,

$$
D\left(\left(\mathbf{g}^{-1} \circ \mathbf{T}_{\omega_{1}}^{-1} \circ \cdots \circ \mathbf{T}_{\omega_{n}}^{-1}\right) \mu_{L}\right) \stackrel{\nu \text {-п.н. }}{\longrightarrow} 0 \quad(n \rightarrow \infty) .
$$

В силу (3) это означает, что

$$
D\left(\left(\mathbf{T}_{\omega_{n}} \circ \cdots \circ \mathbf{T}_{\omega_{1}}\right) \mu\right) \stackrel{\nu \text {-п.н. }}{\longrightarrow} 0 \quad(n \rightarrow \infty) .
$$

Частный случай разобран.

Разберем теперь общий случай. Положим $\mu_{1}=\left(\mu+\mu_{L}\right) / 2$. Тогда $\mu_{1}$ является мерой без атомов, причем носитель ее - вся окружность. Таким образом,

$$
D\left(\left(\mathbf{T}_{\omega_{n}} \circ \cdots \circ \mathbf{T}_{\omega_{1}}\right) \mu_{1}\right) \stackrel{\nu \text {-п.н. }}{\longrightarrow} 0 \quad(n \rightarrow \infty) .
$$

Ho

$$
\left(\mathbf{T}_{\omega_{n}} \circ \cdots \circ \mathbf{T}_{\omega_{1}}\right) \mu_{1}=\frac{\left(\mathbf{T}_{\omega_{n}} \circ \cdots \circ \mathbf{T}_{\omega_{1}}\right) \mu+\left(\mathbf{T}_{\omega_{n}} \circ \cdots \circ \mathbf{T}_{\omega_{1}}\right) \mu_{L}}{2} .
$$

В силу предложения 10 имеем

$$
D\left(\left(\mathbf{T}_{\omega_{n}} \circ \cdots \circ \mathbf{T}_{\omega_{1}}\right) \mu\right) \leqslant 2 D\left(\left(\mathbf{T}_{\omega_{n}} \circ \cdots \circ \mathbf{T}_{\omega_{1}}\right) \mu_{1}\right) .
$$

Следовательно,

$$
D\left(\left(\mathbf{T}_{\omega_{n}} \circ \cdots \circ \mathbf{T}_{\omega_{1}}\right) \mu\right) \stackrel{\nu \text {-п.н. }}{\longrightarrow} 0 \quad(n \rightarrow \infty) .
$$

Теорема 3 доказана.

ПредЛОЖЕНИЕ 11. Пусть $R_{n}$ - последовательность гомеоморфизмов окружсности, $z \in S^{1}$, и пусть $\mathbf{R}_{n} \mu_{L} \rightarrow \delta_{z}$ nри $n \rightarrow \infty$. Тогда для любых $x, y \neq z$

$$
\rho\left(R_{n}^{-1}(x), R_{n}^{-1}(y)\right) \rightarrow 0 \quad(n \rightarrow \infty)
$$

ДокАЗАТЕЛЬСТВО. Поскольку $\mathbf{R}_{n} \mu_{L} \rightarrow \delta_{z}$ при $n \rightarrow \infty$, то

$$
\forall \varepsilon>0 \exists k \in \mathbb{N}: \forall n>k \quad \mathbf{R}_{n} \mu_{L}\left(U_{\varepsilon}(z)\right)>1-\varepsilon .
$$

Но это равносильно тому, что

$$
\forall \varepsilon>0 \exists k \in \mathbb{N}: \forall n>k \quad \mu_{L}\left(R_{n}^{-1}\left(S^{1} \backslash U_{\varepsilon}(z)\right)\right)<\varepsilon .
$$

При $\varepsilon<\min (\rho(z, x), \rho(z, y)) / 2$ обе точки $x$ и $y$ принадлежат дуге $S^{1} \backslash U_{\varepsilon}(z)$. Но прообраз этой дуги под действием $R_{n}$ имеет меру Лебега, а следовательно, и длину, меньшую $\varepsilon$. При $n>k$

$$
\rho\left(R_{n}^{-1}(x), R_{n}^{-1}(y)\right)<\varepsilon .
$$

В силу произвольности $\varepsilon$ при $x, y \neq z$

$$
\rho\left(R_{n}^{-1}(x), R_{n}^{-1}(y)\right) \rightarrow 0 \quad(n \rightarrow \infty) .
$$


ДоКАЗАТЕЛЬСТВо теОРемЫ 1. Заметим, что в силу теоремы 7 в предположениях теоремы 1 при почти всех $\omega$ существует такое $z=z(\omega)$, задаваемое как $f^{+}$для системы $\left(T_{1}^{-1}, \ldots, T_{s}^{-1}\right)$, что

$$
\lim _{n \rightarrow \infty}\left(\mathbf{T}_{\omega_{1}}^{-1} \circ \cdots \circ \mathbf{T}_{\omega_{n}}^{-1}\right) \mu_{L}=\delta_{z} .
$$

Поэтому при почти всех $\omega$ при $x, y \neq z(\omega)$

$$
\begin{aligned}
\lim _{n \rightarrow \infty} \rho\left(\left(T_{\omega_{n}} \circ \cdots \circ T_{\omega_{1}}\right)(x),\left(T_{\omega_{n}} \circ \cdots \circ T_{\omega_{1}}\right)(y)\right) \\
\quad=\lim _{n \rightarrow \infty} \rho\left(\left(T_{\omega_{1}}^{-1} \circ \cdots \circ T_{\omega_{n}}^{-1}\right)^{-1}(x),\left(T_{\omega_{1}}^{-1} \circ \cdots \circ T_{\omega_{n}}^{-1}\right)^{-1}(y)\right)=0 .
\end{aligned}
$$

Несложно видеть, что распределение значений $z(\omega)$ стационарно относительно $T_{i}^{-1}$, взятых с теми же весами $p_{i}$. В силу минимальности системы $\left(S^{1} ; T_{1}^{-1}\right.$, $\left.\ldots, T_{s}^{-1}\right)$ и предложения 6 у этого распределения нет атомов, т. е. $\nu\left\{\omega \mid z(\omega)=z_{0}\right\}$ $=0$ для любого $z_{0} \in S^{1}$.

ДОКАЗАТЕЛЬСТВО СЛЕДСТВИЯ 1 . Для любых двух точек $x$ и $y$ на окружности почти наверное $z(\omega) \neq x, y$, и, следовательно,

$$
\lim _{n \rightarrow \infty} \rho\left(\left(T_{\omega_{n}} \circ \cdots \circ T_{\omega_{1}}\right)(x),\left(T_{\omega_{n}} \circ \cdots \circ T_{\omega_{1}}\right)(y)\right)=0 .
$$

\section{§6. Пример $C^{1}$-открытого множества в пространстве систем, удовлетворяющего предположениям теоремы 1}

Следующий пример принадлежит А. С. Городецкому и Ю. С. Ильяшенко (см. $[10,11])$.

Пусть $T_{1}$ - диффеоморфизм окружности класса $C^{1}$, имеющий притягивающую неподвижную точку $a$ с мультипликатором $T_{1}^{\prime}(a)$, лежащим в интервале $(1 / 2,1)$. Обозначим через $R_{\alpha}$ поворот на угол $\alpha$ (если окружность представлена как $\mathbb{R} / \mathbb{Z}$, то $\left.R_{\alpha}(x)=x+\alpha\right)$. Пусть $\varphi \notin Q$. Рассмотрим отображение $T_{2}=R_{\varphi}$.

ПРеДЛОЖЕНИЕ 12. Существует окрестность $U$ пары $\left(T_{1}, T_{2}\right)$ в пространстве $\operatorname{Diff}^{1}\left(S^{1}\right) \times \operatorname{Diff}^{1}\left(S^{1}\right)$, такал, что любал пара из этой окрестности образует минимальную систему на окружности.

ДокАЗАТЕЛЬСтво. Заметим, что

$$
\exists \delta>0: \forall x \in[a-\delta, a+\delta] \quad 1 / 2<T_{1}^{\prime}(x)<1 .
$$

Пусть $V=(a-\delta, a+\delta)$; обозначим через $C_{V}$ множество диффеоморфизмов окружности, являющихся сжимающими на $\bar{V}$, так что расстояния между точками из $V$ уменьшаются не более чем в два раза,

$$
C_{V}=\left\{g \in \operatorname{Diff}^{1}\left(S^{1}\right) \mid g(\bar{V}) \subset V, \forall x \in \bar{V} 1 / 2<g^{\prime}(x)<1\right\} .
$$

Заметим, что $C_{V}$ открыто в $C^{1}$-топологии. Кроме того, отображение $a: C_{V} \rightarrow V$, сопоставляющее отображению $g \in C_{V}$ его неподвижную точку в $V$, непрерывно в $C^{1}$-топологии.

Из открытости множества $C_{V}$ и того, что $T_{1} \in V$, следует, что

$$
\exists \varepsilon_{0}>0: \forall \alpha, 0<\alpha<\varepsilon_{0} \quad R_{\alpha} \circ T_{1} \in C_{V},
$$

т. е. композиция $T_{1}$ с поворотом на достаточно малый угол принадлежит $C_{V}$. $\mathrm{C}$ другой стороны, в силу иррациональности $\varphi$ найдется степень поворота $T_{2}$, 
являющаяся поворотом на достаточно малый угол:

$$
\exists n: 0<\{n \varphi\}<\varepsilon_{0}
$$

здесь $\{x\}$ - дробная часть $x$. Тогда отображение $g_{2}=T_{2}^{n} \circ T_{1}$ принадлежит $C_{V}$.

Имеет место следующее утверждение: для всех $\tilde{g}_{1}, \tilde{g}_{2} \in C_{V}$ пара $\left(\tilde{g}_{1}, \tilde{g}_{2}\right)$ минимальна на дуге $\gamma=\left[a\left(\tilde{g}_{1}\right), a\left(\tilde{g}_{2}\right)\right]$. Действительно, отображения $\tilde{g}_{1}$ и $\tilde{g}_{2}$ переводят дугу $\gamma$ в себя, причем $\tilde{g}_{1}(\gamma) \cup \tilde{g}_{2}(\gamma)=\gamma$ (последнее следует из того, что никакие расстояния в $\gamma$ больше чем в два раза не уменьшаются). Кроме того, как $\tilde{g}_{1}$, так и $\tilde{g}_{2}$ - сжимающие отображения на $\gamma$. Известно (см. [12]), что если на некотором компакте заданы несколько сжимающих отображений, таких, что объединение их образов совпадает с исходным компактом, то такая система минимальна. Следовательно, $\left(\gamma ; \tilde{g}_{1}, \tilde{g}_{2}\right)$ - минимальная система.

Заметим, что образы открытой дуги $\left(a\left(g_{1}\right), a\left(g_{2}\right)\right)$ под действием итераций иррационального поворота $T_{2}$ покрывают всю окружность; значит, можно выделить конечное подпокрытие $\Omega^{+}$. Кроме того, поскольку отображение $T_{2}^{-1}$ минимально, найдется конечное покрытие $\Omega^{-}$окружности образами дуги $\left(a\left(g_{1}\right), a\left(g_{2}\right)\right)$ под действиями итераций отображения $T_{2}^{-1}$.

Найдется такая окрестность $U_{0}$ пары $\left(T_{1}, T_{2}\right)$, что

$$
\forall\left(\widetilde{T}_{1}, \widetilde{T}_{2}\right) \in U_{0} \quad \widetilde{T}_{1} \in C_{V}, \widetilde{T}_{2}^{n} \circ \widetilde{T}_{1} \in C_{V}
$$

Положим $\tilde{g}_{1}=\widetilde{T}_{1}, \tilde{g}_{2}=\widetilde{T}_{2}^{n} \circ \widetilde{T}_{1}$. Заметим, что концы итераций дуги $\left(a\left(\tilde{g}_{1}\right), a\left(\tilde{g}_{2}\right)\right)$ под действием $\widetilde{T}_{2}$ непрерывно зависят от $\left(\widetilde{T}_{1}, \widetilde{T}_{2}\right)$. Но $\Omega^{+}-$конечное покрытие окружности интервалами; значит, при достаточно малом шевелении этих интервалов оно остается покрытием. Следовательно, найдется такая окрестность $U_{1} \subset U_{0}$, для каждой пары из которой окружность покрывается итерациями дуги $\left(a\left(\tilde{g}_{1}\right), a\left(\tilde{g}_{2}\right)\right)$ под действием $\widetilde{T}_{2}$.

Аналогично найдется такая окрестность $U_{1} \subset U_{0}$, для каждой пары из которой окружность покрывается итерациями дуги $\left(a\left(\tilde{g}_{1}\right), a\left(\tilde{g}_{2}\right)\right)$ под действием $\widetilde{T}_{2}^{-1}$. Покажем, что окрестность $U=U_{0} \cap U_{1} \cap U_{2}$ удовлетворяет условиям предложения.

Действительно, пусть $\left(\widetilde{T}_{1}, \widetilde{T}_{2}\right) \in U$ и некоторое замкнутое непустое множество $M \subset S^{1}$ удовлетворяет условиям $\widetilde{T}_{1}(M) \subset M, \widetilde{T}_{2}(M) \subset M$.

Множество $M$ непусто, а следовательно, существует некоторая точка $x \in M$. Окружность покрывается образами дуги $\gamma=\left[a\left(\tilde{g}_{1}\right), a\left(\tilde{g}_{2}\right)\right]$ под действием $\widetilde{T}_{2}^{-1}$ (поскольку она покрывается образами дуги $\left.\left(a\left(\tilde{g}_{1}\right), a\left(\tilde{g}_{2}\right)\right)\right)$; следовательно,

$$
\exists k \geqslant 0: \quad x \in \widetilde{T}_{2}^{-k}(\widetilde{\gamma}) \Longrightarrow \widetilde{T}_{2}^{k}(x) \in \widetilde{\gamma}
$$

Но $\widetilde{T}_{2}^{k}(x) \in M$, а значит, $M \cap \gamma \neq \varnothing$.

С другой стороны, система $\left(\tilde{g}_{1}, \tilde{g}_{2}\right)$ минимальна на $\gamma$, а $M$ инвариантно относительно $\tilde{g}_{1}$ и $\tilde{g}_{2}$ как композиций $\widetilde{T}_{1}$ и $\widetilde{T}_{2} ;$ следовательно, $M \supset \gamma$.

Наконец, образы дуги $\gamma$ под действием $\widetilde{T}_{2}$ покрывают всю окружность; следовательно, $M=S^{1}$.

Тем самым минимальность произвольной пары $\left(\widetilde{T}_{1}, \widetilde{T}_{2}\right) \in U$ доказана.

Из этого предложения легко следует наличие в пространстве пар отображений с $C^{1}$-топологией на нем открытого множества систем, в котором выполняются предположения теоремы 1. 
Как следствие при всех $k \geqslant 1$ в пространстве пар отображений с $C^{k}$-топологией на нем найдется открытое множество систем, в котором выполняются предположения теоремы 1. То же верно и в отношении топологии $C^{\infty}$.

\section{$\S 7$. Обобщения основных результатов: динамика на других множествах}

Этот параграф посвящен анализу доказательств и описанию возможных обобщений для случайной динамики на других множествах.

Тот факт, что фазовым пространством является окружность - одномерное многообразие - используется при доказательстве основных результатов два раза, в предложении 3 (см. замечание 6) и в предложении 9. Второе использование одномерности - для перехода от одного порядка итераций к другому (предложение 9) - не столь существенно; этот переход может быть проведен другими методами, не использующими одномерность системы.

Однако первая часть предложения 3 существенно опирается на одномерность окружности. В терминах действия полугруппы (порожденной $T_{i}$ ) на фазовом пространстве (окружности) используемое свойство может быть сформулировано следующим образом: для любого элемента g найдется не очень далекий от единицъ элемент $h$, такой, что композиция $g \circ h$ сжимает бо́льшую часть фазового пространства почти в точку. При этом удаление $h$ от единиць определяется лищь необходимой степенью сэсатия - но оно равномерно ограничено no $g$. (Эта часть действительно существенно использует одномерность: ведь мы должны подобрать $h$ так, чтобы применение $g$ после(!) $h$ привело к существенному сжатию.)

Более нигде в доказательствах одномерность ситуации не используется; поэтому всякий раз, когда действие обладает описанным в предыдущем абзаце свойством, для него имеет место сходимость итераций мер к $\delta$-мерам - эффект сжатия в точку (пусть и в несколько ослабленной форме). Это, в частности, означает применимость техники настоящей работы, например, к случаю проективной динамики в предположениях типа общности положения (тем самым передоказывая в чуть более слабой форме упомянутый во введении результат Фюрстенберга [4, Theorem 8.3]). Также техника настоящей работы может быть применена к действию группы линейных преобразований на пространстве флагов, однако, к сожалению, все эти примеры применения не дают результатов, более сильных, чем уже известные. Поэтому мы ограничили подробное изложение случаем одномерной динамики.

\section{§8. Некоторые открытые вопросы}

Теорема 6 гарантирует сходимость итераций с измененным порядком применения отображений для итераций стационарной меры для любого компакта. Для окружности предельная мера в предположениях теоремы 1 (или в предположениях теоремы 5) атомарна.

Однако для многообразий более высокой размерности предельная мера, кажется, имеет гораздо более сложную структуру. Например, для некоторых динамических систем на торе компьютерные эксперименты дают дробную хаусдорфову размерность предельной меры. Интересно было бы исследовать, например, размерность предельной меры. Кроме того, в компьютерных экспериментах мы наблюдаем итерации меры Лебега, а не стационарной меры. Интересно, когда при итерациях меры Лебега предел существует и притом такой же, как и при итерациях стационарной меры. 


\section{ЛитерАТУРА}

1. Kaijser T. On stochastic perturbations of iterations of circle maps. Phys. D, 68, 201-231 (1993).

2. Ширяев А. Н. Вероятность. Наука, М., 1980.

3. Le Jan $Y$. Équilibre statistique pour les produits de difféomorphismes aléatiores indépendants. Ann. Inst. H. Poincaré Probab. Statist., 23, No. 1, 111-120 (1987).

4. Furstenberg H. Noncommuting random matrices products. Trans. Amer. Math. Soc., 108, 377-428 (1963).

5. Furstenberg $H$. Boundary theory and stochastic processes on homogeneous spaces. In: Proc. Sympos. Pure Math., Vol. 26, 1973, pp. 193-229.

6. Furstenberg H., Kesten H. Products of random matrices. Ann. Math. Stat., 31, 457469 (1960).

7. Furstenberg H., Kifer Yu. Random matrix products and measures on projective spaces. Israel J. Math., 46, No. 1-2, 12-32 (1983).

8. Kaimanovich V., Mazur $H$. The Poisson boundary of the mapping class group. Invent. Math, 125, 221-264 (1996).

9. Crauel $H$. Extremal exponents of random dynamical systems do not vanish. J. Dynam. Differential Equations, 2, No. 3, 245-291 (1990).

10. Городецкий А. С., Илъяшенко Ю. С. Некоторые новые грубые свойства инвариантных множеств и аттракторов динамических систем. Функц. анализ и его прил., 33, вып. 2, 16-30 (1999).

11. Городецкий A. С., Ильяшенко Ю. С. Некоторые свойства косых произведений над подковой и соленоидом. Труды МИРАН, 231, 96-118 (2000).

12. Hutchinson J. E. Fractals and self similarity. Indiana Univ. Math. J., 30, 271-280 (1981).

13. Baxendale P. H. Lyapunov exponents and relative entropy for a stochastic flow of diffeomorphisms. Probab. Theory Related Fields, 81, 521-554 (1989).

Московский государственный университет

Независимый московский университет

École Normale Supérieure de Lyon (UMPA 5669 CNRS)

email: kleptsyn@mccme.ru

Московский государственный университет

email: maxim@mccme.ru
Поступило в редакцию

8 мая 2002 г. 